\title{
Quantum Field Theory in the de Sitter Universe
}

\author{
Jacques Bros, ${ }^{1}$ Jean-Pierre Gazeau, ${ }^{2}$ and Ugo Moschella ${ }^{1 *}$ \\ 'Service de Physique Théorique, Centre d'Etudes Nucleaire de Saclay, 9119I Gif-sur-Yvette, France \\ ${ }^{2}$ Laboratoire de Physique Théorique et Mathématique, Université Paris 7, 75251 Paris Cedex 05. France
} (Received 25 May 1994)

\begin{abstract}
We present a study of quantum scalar fields on de Sitter (dS) space-time based on analyticity in the complexified Riemannian manifold; a new expansion of the linear fields in terms of coordinate independent dS plane waves and an explicit form of the propagators at real or imaginary times are given. Our approach controls the thermal properties and the zero-curvature limit of the fields; it provides a general setting for interacting fields, in which some steps are taken: a Källen-Lehmann type formula for complete propagators and a treatment of a class of self-energy diagrams and their resummation.
\end{abstract}

PACS numbers: 04.62.+v, 02.30.Fn, 11.10.Wx. 11.55.Fv

In the absence of a theory for interacting quantized gravity and matter, the study of quantum field theories (QFT's) on a gravitational background has produced a number of interesting results (for a review, see [1]) which culminate in the celebrated Hawking prediction of blackhole evaporation [2]. In this context linear theories on the de Sitter (dS) space-time are probably the most studied example. This is because the dS solution of the cosmological Einstein equations has the same degree of symmetry as the Minkowski solution and it can be seen as a one-parameter deformation of the latter, involving a fundamental length $R$. This length may also be regarded as providing a ( $\mathrm{dS}$ covariant) infrared cutoff for Minkowskian QFT's, whose removal regenerates automatically Poincaré covariance. Furthermore, the interpretation of dS space-time as a universe in exponential expansion in the context of the inflationary cosmological models [3] has lead to renewed interest in dS QFT's. For all these reasons it would be highly desirable that one could dispose of a global dS-Fourier type calculus for describing wave propagation, "particle states," and second quantization on this universe. Here we present a method which is appropriate to this program; this method also sheds a new light on the "preferred representations" of $\mathrm{dS}$ QFT (see [4] and references therein) and on the way they solve the problem of the absence of a true spectral condition [5] which plagues QFT on curved space-time in the general case. Indeed, while it is simple to formalize the requirements of locality (microcausality) and covariance (when it applies) when one tries to quantize a field on a curved space-time, it is generally impossible to formulate any condition on the spectrum of the "energy" operator (even worse, it is impossible to define such a global object). For this reason there are many inequivalent quantum theories for any single field model on a curved spacetime, the quantizations being mostly linked to particular choices of coordinates on the underlying space-time manifold (which induce corresponding frequence splittings).

We will show that, by transporting some familiar notions of complex Minkowski space-time to the complexi- fied dS space-time, one can lift all ambiguities for-dS QFT's and obtain "vacua" which (in spite of their thermal properties) are the exact analogs of Minkowski vacuum representations (the latter appearing as the limit of the former when the curvature tends to zero). In particular, we shall keep from the Minkowskian case the idea that the properties of analytic continuation of the theory in the complexified space-time are directly related to the energy content of the model considered. In the simplest case of linear dS QFT's, these representations (known in the literature $[1,6]$ under the name of "Euclidean vacua"), will be here completely described at real and imaginary times; in particular we shall compute the propagators by introducing an intrinsic (i.e., coordinate independent) plane wave expansion of them. In the second part of this Letter we will show how our approach suggests a general setting for the theory of interacting fields and present a study of the complete propagators.

The dS space-time is visualized as the hyperboloid with equation $\quad X_{R}=\left\{x \in \mathbb{R}^{5}: x^{(0) 2}-x^{(1) 2}-\cdots-x^{(4) 2}=\right.$ $\left.-R^{2}\right\}$. The ambient space $\mathbb{R}^{5}$ is a Minkowski space whose scalar product $x \cdot y=x^{(0)} y^{(0)}-x^{(1)} y^{(1)}-\cdots-x^{(4)} y^{(4)}$ $\left(x^{2}=x \cdot x\right)$ induces the relevant pseudodistance $d$ on $X_{R}$, namely, $\cosh \frac{d(x, y)}{R}=-\frac{x^{\cdot} y}{R^{2}}$. The causality properties in $X_{R}$ are globally expressed by the light-cone ordering; let $V^{+}=\left\{x \in \mathbb{R}^{5}: x^{(0)}>\sqrt{x^{(1) 2}+\cdots+x^{(4) 2}}\right\}$ be the forward light cone in the ambient space; then, for $x, y$ in $X_{R}, x$ is in the future of $y$ provided $x-y$ is a vector in $V^{+}$. The relativity group of the de Sitter space-time is the Lorentz group $\mathrm{SO}_{0}(1,4)$ of the ambient space (dS group). Since the $\mathrm{dS}$ group acts transitively on $X_{R}$, one can distinguish an origin $x_{0}$ in $X_{R}$; we choose $x_{0}=(0,0,0,0, R)$. The $\mathrm{d} S$-Klein-Gordon field equation is written $\square_{R} \phi+\frac{\mu^{2} c^{2}}{\hbar} \phi=02$, where $\square_{R}$ is the d'Alembertian on $X_{R}, \mu$ is a mass parameter, and $c$ is the speed of light. The definition of appropriate $\mathrm{dS}$ plane waves will require the use of a special basis of solutions of this equation [7]. In contrast with the Minkowskian exponentials, these waves are singular on three-dimensional lightlike manifolds and are defined at 
first sight only on (suitable) halves of $X_{R}$ but we shall give below the appropriate $i \epsilon$ prescription to obtain global waves. Here is the relevant definition: let $\xi$ be (any) null vector in $\mathbb{R}^{5}$ (i.e., $\xi^{2}=0$ ); for $x$ in $X_{R}$ such that $x \cdot \xi>0$ (respectively, $x \cdot \xi<0$ ) consider the function $\psi_{+}^{\xi}(x, s)=\left(\frac{\hbar x \cdot \xi}{m c k}\right)^{s}, s \in \mathbb{C}$ [same definition for $\left.\psi_{-}^{\xi}(x, s)\right]$, where $m$ is a mass parameter. One checks that $\square_{R} \psi_{+}^{\xi}(x, s)=\frac{s(3+s)}{R^{2}} \psi_{+}^{\xi}(x, s)$. Physical values of the parameter $s$ are given by $s=-3 / 2+i \nu$ corresponding to the constant $-s(3+s) / R^{2}=\mu^{2} c^{2} / \hbar^{2}$. The interpretation of $\psi_{+}^{\xi}(x, s)$ as $\mathrm{dS}$ plane waves is supported by

$$
x_{R}(\mathrm{x}) \equiv\left(x^{(0)}=R \sinh \frac{\mathrm{x}^{(0)}}{R}, \vec{x}=\left(x^{(1)}, x^{(2)}, x^{(3)}\right)=R \frac{\mathrm{x}}{|\mathrm{x}|} \cosh \frac{\mathrm{x}^{(0)}}{R} \sin \frac{|\mathrm{x}|}{R}, x^{(4)}=R \cosh \frac{\mathrm{x}^{(0)}}{R} \cos \frac{|\mathrm{x}|}{R}\right) .
$$

A quantum field satisfying the dS-Klein-Gordon equation is determined by specifying its two-point Wightman function $\mathcal{W}_{\nu}\left(x_{1}, x_{2}\right)=\left\langle\Omega, \phi\left(x_{1}\right) \phi\left(x_{2}\right) \Omega\right\rangle$. To expand the latter in terms of dS plane waves, we need an extension of those waves to the whole space-time. This problem has its relevant solution in the complexified dS space-time $X_{R}^{(c)}$. There are distinguished domains of $X_{R}^{(c)}$ which are the analogs of the tubes of the complex Minkowski spacetime, namely, $\mathcal{T}^{+}=\mathrm{T}^{+} \cap X_{R}^{(c)}, \mathcal{T}^{-}=\mathrm{T}^{-} \cap X_{R}^{(c)}$. Here $\mathrm{T}^{ \pm}=\mathbb{R}^{5}+i V^{ \pm}$are the forward and backward tubes in the ambient complex Minkowski space-time $\mathbb{C}^{5}$; these are the (standard) analyticity domains of quantum fields satisfying the positivity of the spectrum of the energy operator. Let $C^{+}=\left\{\xi \in \mathbb{R}^{5}: \xi^{2}=0, \xi^{(0)}>0\right\}$; when $z \in \mathcal{T}^{+}$(or $\left.\mathcal{T}^{-}\right)$and $\xi \in C^{+}$the plane waves

$$
\psi^{\xi}(z, s)=\left(\frac{\hbar z \cdot \xi}{m c R}\right)^{s}, \quad s \in \mathbb{C},
$$

are globally defined because in this case the imaginary part of $z \cdot \xi$ does not change sign. These waves allow us to write a new spectral representation for the propagators:

$$
\mathrm{W}_{\nu}\left(z_{1}, z_{2}\right)=c_{\nu} \int_{\gamma}\left(z_{1} \cdot \xi\right)^{-\frac{3}{2}+i \nu}\left(\xi \cdot z_{2}\right)^{-\frac{3}{2}-i \nu} d \mu_{\gamma}(\xi)
$$

valid for $z_{1}, z_{2}$ in $X_{R}^{(c)}$ such that $z_{1} \in \mathcal{T}^{-}, z_{2} \in \mathcal{T}^{+}$; the integration is performed along any complete submanifold $\gamma$ of the cone $C+$ (i.e., any manifold intersecting at least once every generatrix of the cone) with respect to a corresponding measure $d \mu_{\gamma}(\xi)$. A choice of $\gamma$ which has a direct physical meaning is given by the Lorentz invariant mass shells $\gamma_{4}=\left\{\xi \in C^{+}: \xi^{(4)}=m c\right\} \cup\{\xi \in$ $\left.C^{+}: \xi^{(4)}=-m c\right\}$, and $d \mu_{\gamma_{4}}$ is the corresponding Lorentz invariant measure. The function $\mathrm{W}_{\nu}$ is a solution in both variables of the (complex) dS-Klein-Gordon equation which is analytic in the domain $\mathcal{T}_{12}=\left\{\left(z_{1}, z_{2}\right) \in X_{R}^{(c)} \times\right.$ $\left.X_{R}^{(c)}: z_{1} \in \mathcal{T}^{-}, z_{2} \in \mathcal{T}^{+}\right\}$. Furthermore, it is actually a function of the single (dS-invariant) variable $\left(z_{1}-\right.$ $\left.z_{2}\right)^{2}=-2 R^{2}-2 z_{1} \cdot z_{2}$. This property permits an explicit computation, by fixing one of the two points; we obtain that $\mathrm{W}_{\nu}$ is proportional to a Gegenbauer function of the their large $R$ behavior; in fact, parametrizing $\xi$ by the wave vector of a (Minkowskian) particle of mass $m$, i.e., $\xi=\left[\mathrm{k}^{0}, \overrightarrow{\mathrm{k}},-\frac{m c}{\hbar}\right]$, with $\mathrm{k}^{0}=\sqrt{\overrightarrow{\mathrm{k}}^{2}+\frac{m^{2} c^{2}}{\hbar^{2}}}$, and taking $\mu^{2}=m^{2}+\left(\frac{3 \hbar}{2 c R}\right)^{2}$ (i.e., $\nu=m c R / \hbar$ ), we get

$$
\lim _{R \rightarrow \infty}\left(\frac{\hbar x_{R}(\mathrm{x}) \cdot \xi}{m c R}\right)^{-\frac{3}{2}+i \frac{m c R}{\hbar}}=\exp (i \mathrm{k} \cdot \mathrm{x})
$$

here, the dS point $x_{R}(\mathrm{x})$ has to be parametrized by the Minkowskian variable $\mathrm{x} \equiv\left(\mathrm{x}^{(0)}=c t, \overrightarrow{\mathrm{x}}\right)$ measured in units of the $\mathrm{dS}$ radius $R$; for instance,

first kind [8],

$$
\mathrm{W}_{\nu}\left(z_{1}, z_{2}\right)=\frac{2 c_{\nu} e^{\pi \nu} \pi^{2}}{m c R^{3}} P_{-\frac{3}{2}+i \nu}^{(5)}\left(\frac{z_{1} \cdot z_{2}}{R^{2}}\right),
$$

where $c_{\nu}=2 m c^{3} R \Gamma\left(\frac{3}{2}\right)\left(1+4 \nu^{2}\right) e^{-\pi \nu} /(2 \sqrt{\pi})^{7} \cosh \pi \nu$ is obtained by imposing the CCR's. Equations (3) and (4) exhibit the following properties of the two-point function:

(1) $\mathrm{W}_{\nu}\left(z_{1}, z_{2}\right)$ is maximally analytic, i.e., can be analytically continued in the "cut domain" $\Delta=X_{R}^{(c)} \times$ $X_{R}^{(c)} \backslash\left\{\left(z_{1}, z_{2}\right) \in X_{R}^{(c)} \times X_{R}^{(c)}:\left(z_{1}-z_{2}\right)^{2}=\rho \geq 0\right\}$.

(2) $\mathrm{W}_{\nu}\left(z_{1}, z_{2}\right)$ satisfies in $\Delta$ the complex covariance condition: $\mathrm{W}_{\nu}\left(g z_{1}, g z_{2}\right)=\mathrm{W}_{\nu}\left(z_{1}, z_{2}\right)$ for all $g \in \mathrm{SO}_{0}(1,4)^{(c)}$, the complexified of the group $\mathrm{SO}_{0}(1,4)$.

(3) The two-point Wightman function $\mathcal{W}_{\nu}\left(x_{1}, x_{2}\right)=$ $\left\langle\Omega, \phi\left(x_{1}\right) \phi\left(x_{2}\right) \Omega\right\rangle$ is the boundary value of $\mathrm{W}_{\nu}\left(z_{1}, z_{2}\right)$ from $\mathcal{T}_{12}$ and the "permuted Wightman function" $W_{\nu}\left(x_{2}, x_{1}\right)$ is the boundary value of $\mathrm{W}_{\nu}\left(z_{1}, z_{2}\right)$ from the domain $\mathcal{T}_{21}=\left\{\left(z_{1}, z_{2}\right): z_{1} \in \mathcal{T}^{+}, z_{2} \in \mathcal{T}^{-}\right\}$.

(4) The two-point function is positive definite, i.e., $\int_{X_{R} \times X_{R}} W_{\nu}\left(x_{1}, x_{2}\right) \bar{f}\left(x_{1}\right) f\left(x_{2}\right) d x_{1} d x_{2} \geq 0$, for any $f \in C_{0}^{\infty}\left(X_{d}\right), d x$ denoting the dS-invariant measure on $X_{d}$.

(5) The restriction of $\mathrm{W}_{\nu}$ to the sphere $S_{R}\left(z^{(0)}=\right.$ $i y^{(0)}, \vec{z}$ real) yields the Schwinger function $\mathrm{S}_{\nu}\left(z_{1}, z_{2}\right)$; this is permitted since $S_{R} \times S_{R}$ minus the set of coinciding points $z_{1}=z_{2}$ is a subset of $\Delta$.

Properties (1) and (2) characterize $\mathrm{W}_{\nu}$ as being an invariant perikernel [9] on $X_{R}^{(c)}$ with domain $\Delta$. Property (3) allows the explicit construction of the commutator and the Green functions. Moreover, the boundary value of Eq. (3) from $\mathcal{T}_{12}$ gives a new Fourier type representation for the two-point function:

$$
\begin{aligned}
& W_{\nu}\left(x_{1}, x_{2}\right) \\
& =c_{\nu} \int_{\gamma}\left|x_{1} \cdot \xi\right|^{-\frac{3}{2}+i \nu}\left[\theta\left(x_{1} \cdot \xi\right)+e^{i \pi\left(-\frac{3}{2}+i \nu\right)} \theta\left(-x_{1} \cdot \xi\right)\right] \\
& \times\left|x_{2} \cdot \xi\right|^{-\frac{3}{2}-i \nu}\left[\theta\left(x_{2} \cdot \xi\right)+e^{i \pi\left(-\frac{3}{2}-i \nu\right)} \theta\left(-x_{2} \cdot \xi\right)\right] d \mu_{\gamma}(\xi) \text {. }
\end{aligned}
$$

We stress that Eq. (5) allows a factorization of the twopoint function in terms of (global) plane waves on $X_{R}$ 
which is completely similar to the corresponding Fourier representation for Minkowski fields $\phi$. The latter is recovered as the flat limit of Eq. (5). By proceeding as before we obtain that $\lim _{R \rightarrow x} \mathcal{W}_{m \cdot k}\left(x_{R}(\mathrm{x}), y_{R}(\mathrm{y})\right)=$ $\frac{\hbar c^{2}}{2(2 \pi)^{3}} \int e^{-i \mathrm{k}(\mathrm{x}-\mathrm{y})} \frac{d^{3} \mathrm{k}}{\mathrm{k}^{0}}=\langle\underline{\Omega}, \underline{\phi}(\mathrm{x}) \underline{\phi}(\mathrm{y}) \underline{\Omega}\rangle^{\frac{1}{\hbar}}$. Equation (5) is also used to prove the positivity property (4) and therefore to construct the Fock space of $\phi$, generated by a "oneparticle" space which carries a unitary irreducible representation (of the principal series) of the dS group. We remark that the representations of the Poincaré group obtained as contractions of those irreducible ones of the dS group are not irreducible [10] while representations associated to the fields $\phi$ are. Therefore group-theoretical contraction is not a suitable procedure to draw consequences on the structure of the limiting quantum field theory; in particular, no problem of negative energy arises in the latter. Finally, property (5) makes possible the identification of our models with the "Euclidean vacua" of [6], but here we have exhibited their full analytic structure in real or complex space.

We now give the physical interpretation of the analyticity property (1) of $\mathrm{W}_{\nu}$ : as in [6] we adopt the viewpoint of an observer sitting on the geodesic $h\left(x_{0}\right)$ of $x_{0}$, contained in the $\left(x^{(0)}, x^{(3)}\right)$ plane. The set of all events of $X_{R}$ which can be connected with the observer by the reception and the emission of light signals is the region $U=\left\{x \in X: \quad x^{(3)}>\left|x^{(0)}\right|\right\}$; it is bordered by the "future" and "past" event horizons of the observer $x^{(0)}= \pm x^{(3)}, x^{(3)}>0$. The region $U$ is foliated by hyperbolic trajectories $h_{\underline{x}}\left(x_{0}\right)$ parallel to the geodesic $h\left(x_{0}\right)=h_{0}\left(x_{0}\right)$, according to the following parametrization of $\mathcal{U}: x(\tau, \underline{\vec{x}})=\left(x^{(0)}=\sqrt{R^{2}-\underline{\vec{x}}^{2}} \sinh \frac{c \tau}{R}, \vec{x}=\underline{\vec{x}}\right.$, $\left.x^{(4)}=\sqrt{R^{2}-\underline{x}^{2}} \cosh \frac{c \tau}{R}\right) ; \tau$ is the proper time of our observer. The curves $h_{\underline{\vec{x}}}\left(x_{0}\right)$ are the orbits of the one-parameter group $T_{h\left(x_{0}\right)}$ of isometries of $\mathcal{U}$ (see [4] for a general discussion of this kind of structure): $\quad T_{h\left(x_{0}\right)}(t)[x(\tau, \underline{\vec{x}})]=x(t+\tau, \underline{\vec{x}}) \equiv x^{t}, t \in \mathbb{R}$. The complexified orbits of $T_{h\left(x_{0}\right)}$, namely, the complex hyperbolas $h_{\vec{x}}^{(c)}\left(x_{0}\right)$ have $2 i \pi R / c$ periodicity in $t$ and all their nonreal points in $\mathcal{T}^{+}$and $\mathcal{T}^{-}$. This entails a remarkable property of the time-translated correlation functions $\left\langle\Omega, \phi\left(x_{1}\right) \phi\left(x_{2}^{t}\right) \Omega\right\rangle=\mathcal{W}_{\nu}\left(x_{1}, x_{2}^{t}\right)$ and $\left\langle\Omega, \phi\left(x_{2}^{t}\right) \phi\left(x_{1}\right) \Omega\right\rangle=\mathcal{W}_{\nu}\left(x_{2}^{t}, x_{1}\right)$, where $x_{1}$ and $x_{2}$ are arbitrary events in $\mathcal{U}$. In fact, from the analyticity properties (1) of $\mathrm{W}_{\nu}\left(z_{1}, z_{2}\right)$, we deduce that $\mathrm{W}_{\nu}\left(x_{1}, x_{2}^{t}\right)$ defines a $2 i \pi R / c$ periodic analytic function of $t$, whose domain is the periodic cut plane $\mathbb{C}_{x_{1}, x_{2}}^{\text {cut }}=\{t \in \mathbb{C} ; \Im t$ $\neq 2 n \pi R / c, n \in \mathbb{Z}\} \cup\left\{t ; t-2\right.$ in $\left.\pi R / c \in I_{x_{1}, x_{2}}, n \in \mathbb{Z}\right\}$, where $I_{x_{1}, x_{2}}$ is the real interval on which $\left(x_{1}-x_{2}^{t}\right)^{2}<0$. One also checks that the boundary values of $\mathrm{W}_{\nu}\left(x_{1}, x_{2}^{t}\right)$ on $\mathbb{R}$ coincide with the previous correlation functions (the jumps across the cuts being the retarded and advanced commutators); these properties imply that $\mathrm{W}_{\nu}\left(x_{1}, x_{2}^{t}\right)$ is analytic in the strip $\{t \in \mathbb{C}, 0<\Im t<2 i \pi R / c\}$ and satisfies the following KMS relation [11] at temperature $T=\hbar c / 2 \pi K R$ ( $K$ is the Boltzmann constant):

$$
\mathcal{W}_{\nu}\left(x_{2}^{t}, x_{1}\right)=\lim _{\epsilon \longrightarrow 0} \mathrm{~W}_{\nu^{\prime}}\left(x_{1}, x_{2}^{t+\frac{2 \pi k k}{t}-l \epsilon}\right) . \quad t \in \mathbb{R} .
$$

The "energy operator" $\mathcal{E}_{h\left(x_{0}\right)}$ associated with the geodesic $h\left(x_{0}\right)$ is obtained by the spectral decomposition of the unitary representation of the time translation group $T_{h\left(x_{0}\right)}$ in the Hilbert space $\mathcal{H}$ of the theory, namely, $\quad U_{h\left(x_{0}\right)}^{t}=\int_{-x}^{x} e^{\frac{1}{\hbar} \omega t} d E_{h\left(x_{0}\right)}(\omega)$, which yields $\mathcal{E}_{h\left(x_{0}\right)}=\int_{-x}^{x_{\infty}} \omega d E_{h\left(x_{0}\right)}(\omega)$. The previous KMS condition is then equivalent to the fact that energy measurements performed by an observer at rest at the origin on states localized in $\mathcal{U}$ are exponentially damped by a factor $\exp (-2 \pi R \omega / \hbar c)$ in the range of negative energies. In the limit of flat space-time this factor will kill all negative energies, so that one recovers the usual spectral condition of "positivity of the energy." Similar results concerning the existence of QFT's satisfying the KMS condition have been obtained for the two-dimensional dS universe in [12] by the methods of constructive QFT. Other examples of curved space-time QFT's satisfying the KMS condition have been investigated in [13] where an axiomatic approach has been proposed to study such theories.

Here we shall show how our approach, based on analyticity properties, opens the way to a general setting of the theory of interacting fields on $\mathrm{dS}$ space-time. In short, a scalar field $\phi(x)$ on $X_{R}$ may be assumed to satisfy the following properties (besides positive definiteness):

(a) Locality: $\left[\phi\left(x_{1}\right), \phi\left(x_{2}\right)\right]=0$ for all spacelike separated events $\left(x_{1}, x_{2}\right)$ on $X_{R}$ [i.e., $\left(x_{1}-x_{2}\right)^{2}<0$ ].

(b) $d S$ covariance: There is a unitary representation $U(\cdot)$ of $\mathrm{SO}_{0}(1,4)$ in $\mathcal{H}$ such that $\phi(g x)=U(g) \phi(x) U(g)^{-1}$.

(c) Geodesical spectral condition or geometrical KMS condition: The subgroups of time translations $T_{h}$ of $\mathrm{SO}_{0}(1,4)$ associated with any timelike geodesic $h$ have energy spectrum densities which violate the positivity condition by appropriate exponential damping factors. This is equivalent to the fact that the propagators of $\phi$ (and of any associated polynomial composite field) are analytic in the complexified geodesic $h^{(c)}$ and in all the parallel complex hyperbolic trajectories, so that the corresponding KMS condition (6) is valid. These properties imply that the propagator of the field $\phi$ is an analytic function $\mathrm{W}\left(z, z^{\prime}\right)$ defined in the cut domain $\Delta$, and that it admits the following integral representation $[9,14]$ :

$$
\mathrm{W}\left(z_{1}, z_{2}\right)=\int_{0}^{\infty} \rho(\nu) \mathrm{W}_{\nu}\left(z_{1}, z_{2}\right) d \nu .
$$

with $\rho$ denoting a positive measure. Formula (7), which is the analog of the Källen-Lehmann representation for the propagators of local fields in Minkowski space, relies on a suitable mode expansion in the complexified "mass" variable $\nu=m c R / \hbar$ associated with the global plane waves $\psi^{\xi}\left(z,-\frac{3}{2}+i \nu\right)$. Consider indeed for $\Im \nu \leq 0$ the following Laplace type transform of the retarded function $\Delta_{r} \mathrm{~W}\left(x_{1}, x_{2}\right)=\theta\left(x_{1}^{(0)}, x_{2}^{(0)}\right)\left[\mathcal{W}\left(x_{1}, x_{2}\right)-\mathcal{W}\left(x_{2}, x_{1}\right)\right]$ :

$$
\mathrm{G}(\nu)=\frac{\pi^{2}}{3} \int_{1}^{x} \Delta_{r} \mathrm{~W}(\lambda) Q_{-2+1}^{(5)}(\lambda)\left(\lambda^{2}-1\right) d \lambda
$$


with $-\lambda=x_{1} \cdot x_{2} / R^{2}, Q_{-\frac{3}{2}+i \nu}^{(5)}$ being proportional to a Gegenbauer function of the second kind [8]. One checks that the linear field propagators $\mathrm{W}_{\nu_{0}}\left(z_{1}, z_{2}\right)$ are represented by pole terms of the form $\mathrm{G}_{\nu_{0}}(\nu)=\frac{\nu}{\nu^{2}-\nu_{0}^{2}}$ (considered for $\Im \nu \leq 0$ ) and it follows that any general propagator $\mathrm{W}\left(z_{1}, z_{2}\right)$ is represented by an analytic function $\mathrm{G}$ satisfying a dispersion relation of the form $\mathrm{G}(\nu)=$ $\int_{0}^{\infty} \rho\left(\nu_{0}\right) \mathrm{G}_{\nu_{0}}(\nu) d \nu_{0}$, which, by going back to the spacetime $X_{R}$, yields the representation (7). The interest of this "complex mass formalism" is that it produces a generalized version of the frequency analysis of thermal field theories. As in the latter our propagators $\mathrm{W}\left(z_{1}, z_{2}\right)$ can be considered (in view of their analyticity properties) either in the real time formalism (RTF), i.e., through their relevant boundary values on $X_{R}$, or in the imaginary time formalism (ITF), i.e., on the Euclidean sphere $S_{R}$. Consider the discrete mode expansion of the Schwinger propagator $\mathrm{S}$ [i.e., $\mathrm{W}\left(z_{1}, z_{2}\right)$ for Euclidean $z_{1}, z_{2}$ ] in terms of Gegenbauer polynomials [8]:

$$
\mathrm{S}\left(z_{1}, z_{2}\right)=\frac{1}{16 \pi^{2}} \sum_{n=0}^{\infty} w_{n} c_{n} P_{n}^{(5)}\left(-\frac{z_{1} \cdot z_{2}}{R^{2}}\right),
$$

with $c_{n}=(2 n+3)(n+2)(n+1)$. As in the Matsubara type formalism [15] of thermal QFT, the coefficients $w_{n}$ are shown $[9,14]$ to coincide with the Green function $G$ at imaginary integers, i.e., $w_{n}=\mathrm{G}(-i n)$ (corresponding to discrete imaginary masses $m_{n}=-i n \hbar / R c$ ).

Let us now indicate in which way the previous analytic approach can lead to a sensible perturbation calculus on dS space-time. We shall stick here to a class of selfenergy diagrams which is closed under the operation of line insertion and contraction [see Fig. 1(a)], but the formalism is generalizable to $n$-point Green functions. It is clear that any pointwise product of propagators $\prod_{i=1}^{N} \mathrm{~W}^{(i)}\left(z_{1}, z_{2}\right)$ satisfies the same analytic structure as any individual factor $\mathrm{W}^{(i)}\left(z_{1}, z_{2}\right)$; this rules the case of the contraction operation [Fig. 1(b)]. Let us introduce the line-insertion operation as a convolution on the Euclidean sphere $S_{R}$; for example, for the case of Fig. 1(c), the propagator is

$$
\mathrm{S}\left(z_{1}, z_{2}\right)=\int_{S_{R} \times S_{R}}\left[\mathrm{~S}_{\nu}\left(z_{1}, z\right)\right]^{2} \mathrm{~S}_{\nu}\left(z, z^{\prime}\right)\left[\mathrm{S}_{\nu}\left(z^{\prime}, z_{2}\right)\right]^{2} d \mu d \mu^{\prime} .
$$

One can show by contour distortion [9] that the resulting propagators enjoy the full analytic structure on $X_{R}^{(c)}$ and that the "real-time" counterparts of this class of Euclidean equations must be written in terms of retarded

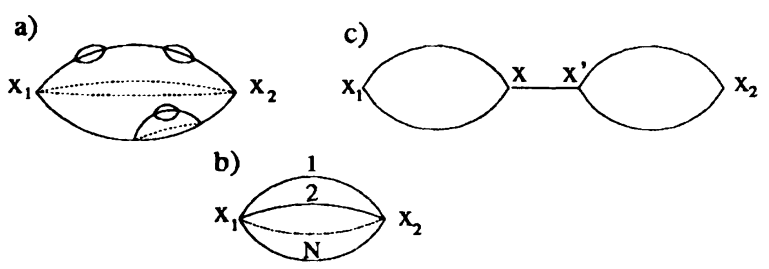

FIG. 1. Self-energy diagrams. propagators. Moreover, as in the Minkowskian case these convolutions are turned into ordinary products of the corresponding Green functions $\mathrm{G}(\nu)$. It follows that one can make the resummation of the self-energy graphs of this class by geometrical series in the complex "mass" variable $\nu$, and that the possible generation of poles is still interpretable in terms of mass renormalization.

To conclude, we wish to stress the importance of global analyticity properties in constructing QFT's on the dS universe; these properties have appeared to be crucial from both computational and conceptual viewpoints. All the QFT's considered have a thermal interpretation in view of the existence of a temporal curvature of a very specific nature (as in the Unruh effect $[13,16]$ and in the black-hole evaporation $[2,13])$; in fact the existence of complex hyperbolic trajectories on which maximal analyticity properties hold consistently with locality is a geometric criterion for QFT "vacua" in which thermal effects are produced, the temperature being proportional to the curvature of these trajectories. This geometrical property, which replaces the spectral condition of the flat case, should be implementable on a wider class of spacetime manifolds admitting a global complexified structure.

U.M. is a EEC Human Capital and Mobility fellow.

*To whom correspondence should be addressed.

[1] N.D. Birrell and P.C.W. Davies, Quantum Fields in Curved Space (Cambridge University Press, Cambridge, 1982).

[2] S. W. Hawking, Commun. Math. Phys. 43, 199 (1975).

[3] A. Linde, Particle Physics and Inflationary Cosmology (Harwood Academic Publishers, Chur, 1990).

[4] B. S. Kay and R. Wald, Phys. Rep. 207, 49 (1991).

[5] R.F. Streater and A.S. Wightman PCT, Spin and Statistics, and All That (W.A. Benjamin, New York, 1964).

[6] G. W. Gibbons and S. W. Hawking, Phys. Rev. D 15, 2738 (1977).

[7] S. Molchanov, Math. Dokl. 7, 1553 (1966).

[8] $P_{-3 / 2+i \nu}^{(5)}(w)$ is proportional to a Gegenbauer function of the first kind $C_{-3 / 2+i \nu}^{(3 / 2)}(w) ; H$. Bateman, Higher Transcendental Functions I (McGraw-Hill, New York, 1954).

[9] J. Bros, Nucl.Phys. (Proc. Suppl.) B18, 22 (1990); J. Bros and G. A. Viano, Saclay Report No. T93-039, 1993 (to be published); Bull. Soc. Math. France 120, 169 (1992).

[10] J. Mickelsson and J. Niederle, Commun. Math. Phys. 27, 199 (1972).

[11] R. Haag, Local Quantum Physics, Fields, Particles, Algebras (Springer, Berlin, 1992).

[12] R. Figari, R. Höegh-Krohn, and C. R. Nappi, Commun. Math. Phys. 44, 265 (1975).

[13] G. L. Sewell, Ann. Phys. (N.Y.) 141, 201 (1982).

[14] J. Bros and U. Moschella, Saclay report (to be published).

[15] See, e.g., N. P. Landsman and T. G. van Veert, Phys. Rep. 145, 142 (1987), and references therein.

[16] W. G. Unruh, Phys. Rev. D 14, 870 (1976). 